Revue d'histoire de l'Amérique française

REVUE D.HISTOIRE DE L'AMÉRIQUE FRANÇAISE

\title{
Analyse de statistiques historiques sur le lectorat du quotidien québécois Le Devoir de 1910 à 2000
}

\section{Marie-Eve Carignan et Claude Martin}

Volume 70, numéro 3, hiver 2017

URI : https://id.erudit.org/iderudit/1039518ar

DOI : https://doi.org/10.7202/1039518ar

Aller au sommaire du numéro

Éditeur(s)

Institut d'histoire de l'Amérique française

ISSN

0035-2357 (imprimé)

1492-1383 (numérique)

Découvrir la revue

Citer cet article

Carignan, M.-E. \& Martin, C. (2017). Analyse de statistiques historiques sur le lectorat du quotidien québécois Le Devoir de 1910 à 2000. Revue d'histoire de l'Amérique française, 70(3), 55-79. https://doi.org/10.7202/1039518ar
Résumé de l'article

Le quotidien montréalais Le Devoir, fondé en 1910 comme un journal indépendant, nationaliste et catholique, a joué un rôle important dans l'évolution de la société québécoise. Selon plusieurs, il fut surtout lu par l'élite sociale et économique du Canada français, puis du Québec. Nous analysons ici l'importance chiffrée du lectorat du Devoir et certaines de ses caractéristiques en nous basant sur les données de tirage de ce quotidien et d'autres journaux. Nous regardons aussi la distribution géographique du journal ainsi que les résultats de certaines enquêtes auprès de ses lecteurs et de l'élite canadienne-française. L'analyse révèle que Le Devoir représente une part relativement stable du tirage des grands journaux en français à Montréal, mais que son lectorat hors du Québec a fortement diminué avec le temps. Son lectorat comporte bien une composante de l'élite québécoise, mais aussi d'autres classes de la société.
Tous droits réservés @ Institut d’histoire de l’Amérique française, 2017
Ce document est protégé par la loi sur le droit d'auteur. L'utilisation des services d'Érudit (y compris la reproduction) est assujettie à sa politique d'utilisation que vous pouvez consulter en ligne.

https://apropos.erudit.org/fr/usagers/politique-dutilisation/ 


\title{
Analyse de statistiques historiques sur le lectorat du quotidien québécois Le Devoir de I9IO à 2000
}

\author{
Marie-Eve Carignan \\ Université de Sherbrooke \\ Claude Martin \\ Université de Montréal et Université du Québec à Trois-Rivières
}

RÉSUMÉ • Le quotidien montréalais Le Devoir, fondé en 1910 comme un journal indépendant, nationaliste et catholique, a joué un rôle important dans l'évolution de la société québécoise. Selon plusieurs, il fut surtout lu par l'élite sociale et économique du Canada français, puis du Québec. Nous analysons ici l'importance chiffrée du lectorat du Devoir et certaines de ses caractéristiques en nous basant sur les données de tirage de ce quotidien et d'autres journaux. Nous regardons aussi la distribution géographique du journal ainsi que les résultats de certaines enquêtes auprès de ses lecteurs et de l'élite canadienne-française. L'analyse révèle que Le Devoir représente une part relativement stable du tirage des grands journaux en français à Montréal, mais que son lectorat hors du Québec a fortement diminué avec le temps. Son lectorat comporte bien une composante de l'élite québécoise, mais aussi d'autres classes de la société.

ABSTRACT - The French-language daily newspaper Le Devoir was founded in Montréal in 1910 as an independent, nationalist and catholic newspaper, and it has played a major part in the development of Québec's society. Many see it as the newspaper of a French Canadian and, later, Québécois social and economic elite. In this article, we analyze the circulation data of Le Devoir and of its main competitors as well as some of its characteristics. We also take a look at its geographic distribution and at survey results pertaining to its readers and other French-Canadian elite. We conclude that Le Devoir holds a relatively constant share of the major French-language newspaper market in Montréal but that its circulation outside of Québec has strongly diminished over time. While the Québécois elite feature strongly in its readership, other social classes are also represented. 
T e journal quotidien montréalais Le Devoir occupe une place particuLlière dans l'histoire de la presse canadienne et québécoise. Fondé en 1910 comme un journal de combat nationaliste et catholique, alors que l'époque est celle du développement des journaux populaires d'information, il s'engage «à défendre les idées et les causes qui favorisent l'avancement politique, économique, culturel et social du Québec ${ }^{1}$ ». Bien que respecté par plusieurs pour son intégrité, il ne sera pas un journal populaire, on le qualifie même d'élitiste ${ }^{2}$. Son tirage et ses finances seront plutôt modestes. Nous voulons analyser ici un aspect particulier de son histoire, soit l'évolution et les caractéristiques de son lectorat, ce qui fait l'originalité de cet article.

\section{LA FONDATION ET L'ÉVOLUTION DU JOURNAL LE DEVOIR}

Le 10 janvier 1910, alors que Montréal compte déjà huit quotidiens, Henri Bourassa fonde Le Devoir. Il avait, à ce moment, «souhaité que son journal demeure totalement indépendant et qu'il ne puisse être vendu à aucun groupe $^{3}$ ». À sa création, ce journal en fut d'abord un «de combat ${ }^{4}$ », comme le sous-entend cette citation de Bourassa dans le premier numéro du quotidien: «Le Devoir appuiera les honnêtes gens et dénoncera les coquins. » Concrètement, Le Devoir sera alors nationaliste et catholique, mais tout de même indépendant de l'Église. Ce journal est aujourd'hui imprimé et publié à Montréal six jours par semaine, en plus de diffuser son contenu sur ses plateformes en ligne et par le moyen d'une «application " pour tablettes électroniques et appareils mobiles.

La notion d'indépendance du journal mérite une explication. D’un point de vue juridique ou économique, une entreprise est indépendante si aucune autre ne possède la majorité des droits de vote conférés par la propriété d'actions émise par cette entreprise. De ce point de vue, Le Devoir est bien un journal indépendant, contrairement à La Presse et au Journal de Montréal qui appartiennent à des conglomérats. Dans le cas d'un média d'information, cette caractéristique est importante, car on peut soupçonner, à tort ou à raison, que le traitement de l’information pourrait

1. Jean-François Nadeau, Le Devoir: Un siècle québécois (Montréal, Les Éditions de l’Homme, 2010), extrait de la quatrième de couverture.

2. Claude Lagadec, "Un journal élitiste peut-il être indépendant?», dans Robert Comeau et Luc Desrochers, dir., Le Devoir. Un journal indépendant (1910-1995) (Québec, Presses de l’Université du Québec, 1996).

3. Le Devoir, "Qui sommes-nous», www.ledevoir.com/le-devoir/le-devoir [consulté le 16 novembre 2016].

4. Id. 
être influencé par la propriété du journal ${ }^{5}$. Lors de la création du Devoir, le journal avait bien des actionnaires, mais ceux-ci ne cherchaient pas une rentabilité pour leur investissement ni une influence directe sur le contenu du journal. Ils visaient justement un journal indépendant. C'est pourquoi le fondateur, les actionnaires et les administrateurs du Devoir ont développé une structure juridique qui donnait au directeur tous les pouvoirs. Selon Sylvio Normand, «L'indépendance du directeur devient le leitmotiv qui l'emporte sur les autres considérations. La structure juridique imaginée par les fondateurs permet d'assurer la pérennité de cette finalité6." Cette structure juridique a cependant connu quelques limites lors de la nomination d'un nouveau directeur. Par exemple, en 1946, les pouvoirs d'une fiducie impliquée dans la nomination d'un directeur sont transférés à l'archevêque de Montréal, $\mathrm{M}^{\mathrm{gr}} \mathrm{J}$ Joseph Charbonneau ${ }^{7}$. À ces occasions et lors d'épisodes de fragilité financière, on voit ainsi se profiler un réseau d'appuis au journal, ce que certains considèrent, encore à tort ou à raison, comme une influence sur le quotidien. En 1992, le Fonds de solidarité des travailleurs du Québec, le Fonds de coopération Desjardins et les employés du journal deviennent actionnaires ${ }^{8}$, mais le directeur conserve encore tous les droits de gestion. On peut aussi simplement voir ce réseau comme une saine manifestation du lien entre le journal et la société québécoise. Ainsi, Le Devoir demeure un journal indépendant.

Le Devoir se voit souvent qualifié de journal cherchant à rejoindre un lectorat représentant l'élite canadienne-française, mais les perceptions sur les intérêts de ses lecteurs et sur ses principaux compétiteurs dans l'univers des médias varient d'un observateur à l'autre. Ainsi, Jean-François Nadeau affirme que, dès sa fondation, Le Devoir n'a "pas de frontière politique au sens strict» et qu'il "comprend en fait toute la diaspora canadienne-française et englobe aussi bien la Nouvelle-Angleterre que l'ensemble du territoire canadien, d'un océan à l'autre " ${ }^{9}$. Selon lui, le journal est dis-

5. Voir notamment à ce sujet: Marc-François Bernier, Journalistes au pays de la convergence. Sérénité, malaise et détresse dans la profession (Québec, Les Presses de l'Université Laval, 2008); Marc-François Bernier, Rapport d'analyse de l'enquête quantitative sur l'indépendance journalistique, Document exclusif présenté au Conseil de presse du Québec (Ottawa, février 2014) et Marc-François Bernier, «L'indépendance journalistique sous pression dans les conglomérats médiatiques québécois», dans Éric George, dir., Concentration des médias, changements technologiques et pluralisme de l'information (Québec, Les Presses de l’Université Laval, 2015), 111-135.

6. Sylvio Normand, "La structure juridique du journal Le Devoir», Communication, 29, 2 (2012), en ligne, http:/ / communication.revues.org/2748.

7. Ibid., 6.

8. Ibid., 10.

9. J.-F. Nadeau, op. cit., «Chapitre 2: Défense 1920-1930», 52. 
tribué largement dans le but de "défendre la langue française et sa culture", de "renforcer le sentiment d'appartenance à la communauté canadienne-française et à la religion catholique», mais il a aussi pour objectif de "susciter des abonnements »"

Comme nous le verrons plus loin, plusieurs journaux surpassent Le Devoir par leur tirage, en particulier le quotidien La Presse qui dominera longtemps le paysage montréalais sur cet aspect. Cependant, revenant sur l'histoire du Devoir, alors qu'il fêtait son $75^{\mathrm{e}}$ anniversaire de fondation en 1985, Pierre-Philippe Gingras estime pour sa part que la compétition la plus menaçante du journal ne venait "pas des autres quotidiens francophones de Montréal, mais plutôt du seul journal quotidien anglophone de la métropole, The Gazette» qui lui livrait alors «une lutte serrée au plan surtout des pages économiques et financières » ${ }^{11}$. Interrogé par Gingras, Jean-Louis Roy, alors directeur du journal, estimait que les nouveaux lecteurs se recrutaient alors "parmi des groupes francophones qui lisaient The Gazette surtout pour ses colonnes économiques», mais que le journal comptait également «une nouvelle clientèle issue de groupes des communautés culturelles qui, dans la logique de leur inscription au Québec dans les années 1960 et au début de la décennie suivante» découvraient maintenant qu'ils pouvaient «être correctement informés de façon substantielle et fiable par Le Devoir»" ${ }^{12}$. Pour Roy, Le Devoir se situait «à part sur le marché des quotidiens francophones de Montréal ${ }^{13}$ » et n'avait pas l'intention d'investir les marchés de la presse populaire principalement occupés par le Journal de Montréal et La Presse.

Cependant, 25 ans plus tard, à l'occasion des 100 ans du quotidien, Stéphane Baillargeon indique que Le Devoir s'inscrit dans une réalité de marché où il doit un peu faire comme les autres journaux d'information pour survivre, «tout en développant son propre créneau ${ }^{14}$ ». Il ajoute que le défi du quotidien, à l'aube de son deuxième siècle d'existence, est de rejoindre le public et la nouvelle génération de lecteurs sur Internet et de s'adapter à la multiplication des sources d'information. Thierry Watine abonde dans le même sens lorsqu'il affirme que, dans un souci «d'élargir un lectorat insuffisant (environ 30000 exemplaires par jour en 2000), Le

10. Id.

11. Pierre-Philippe Gingras, Le Devoir (Montréal, Libre Expression, 1985), 286.

12. Ibid., 286-287.

13. Ibid., 287.

14. Fernande Roy en entrevue avec Stéphane Baillargeon, «1910: Henri Bourassa crée Le Devoir pour combler un vide dans l'offre de l'information", Le Devoir, édition des samedi 9 et dimanche 10 janvier 2010, page A-2. 
Devoir a manifestement ouvert les vannes d'une approche de plus en plus marketing où la forme tend à rivaliser avec le fond ${ }^{15}$ " et où les dirigeants du journal cherchent «à élargir leur assiette de lecteurs, quitte à remiser quelques vieux principes journalistiques ${ }^{16} »$.

\section{NOS DONNÉES, NOTRE MÉTHODE}

Dans le champ des études en communication, le problème de la réception des messages médiatiques a fait l'objet de nombreuses recherches, en particulier sous l'aspect de l'influence des médias, mais aussi en considérant l'utilité de ceux-ci pour les auditoires. Les recherches sur les auditoires des médias font appel à une palette de méthodes quantitatives et qualitatives. Les approches quantitatives se prêtent bien à des questions comme la détermination du nombre de lecteurs d'un journal ou les caractéristiques sociales du lectorat. Les approches qualitatives sont adaptées à des recherches sur les modalités de la lecture ou sur les motivations du lectorat. Les deux approches peuvent se combiner dans le but de mieux comprendre un phénomène ${ }^{17}$, mais si nous voulons remonter le temps et procéder à une analyse historique, la disponibilité des données ne sera pas la même que dans le cas d'une recherche contemporaine.

Au départ, notre recherche sur le lectorat du Devoir a été rendue possible par l'existence d'une base de données sur les tirages des périodiques québécois entre 1850 et 2003 constituée et mise à notre disposition par le Groupe de recherche sur les mutations du journalisme (GRMJ) de l'Université Laval, groupe alors dirigé par Jean Charron et Jean de Bonville. Ces données ont été compilées à partir de plusieurs sources dont, en particulier, des répertoires de données destinées aux agences de publicité ${ }^{18}$. Dans ces répertoires, les données de tirage des périodiques étaient présentées systématiquement et, surtout, dès le début du $\mathrm{XX}^{\mathrm{e}}$ siècle, leur exactitude était vérifiée, car le prix d'une annonce est d'abord fonction du nombre d'exemplaires vendus. De plus, le GRMJ a mis à notre disposition certains documents tirés des archives du Devoir, soit quatre enquêtes

15. Thierry Watine, «Le Devoir est-il encore un journal de référence?», Les Cahiers du journalisme, 7 (décembre 2000): 43.

16. Ibid., 44.

17. Par exemple, dans Denis Saint-Jacques, Jacques Lemieux, Claude Martin et Vincent Nadeau, Ces livres que vous avez aimés. Les best-sellers au Québec de 1970 à aujourd'hui, 2éd. Révisée (Québec, Nuit Blanche Éditeur, 1997).

18. Groupe de recherche sur les mutations du journalisme (GRMJ), Hiperbec: la banque des journaux et les périodiques québécois, en ligne, www.com.ulaval.ca/recherche/groupes-de-recherche/groupe-derecherche-sur-les-mutations-du-journalisme-grmj/banques-de-donnees/hiperbec-la-banque-des-journaux-et-les-periodiques-quebecois/ [consulté le 29 février 2016]. 
réalisées entre 1942 et 1984 ainsi qu'une lettre de 1954, fort intéressante. Nous ferons aussi appel à une enquête de 1944 sur les comportements médiatiques des Canadiens français.

Dans notre démarche, le journal est analysé comme une organisation ou une entreprise. C'est cette organisation même qui est soumise à l'analyse historique. Cela diffère de l'usage le plus fréquent du journal en histoire, soit celui d'être une source d'archives pour l'analyse d'événements historiques. En 2000, Fernande Roy et Jean de Bonville appelaient de leurs vœux le développement d'une telle approche méthodologique: «L'histoire de la presse ne progressera vraiment que lorsque les historiens considéreront la presse en elle-même et pour elle-même, et non plus simplement comme une source commode de renseignement ${ }^{19}$.» Roy et de Bonville proposaient des «orientations de recherche» susceptibles de combler les "profondes lacunes» observées dans l'historiographie ${ }^{20}$. En particulier, ils définissaient un champ de recherche sur le lectorat ${ }^{21}$. Dominique Marquis, dans une publication plus récente, se désolait également du peu de développement de ce champ d'études: "La majorité de ces travaux [des historiens] s'intéressent toujours au seul contenu de la presse", observait-elle ${ }^{22}$. C'est à ce domaine encore lacunaire de l'histoire de la presse et de son lectorat que le présent texte s'intéresse.

Notre travail consiste d'abord à analyser certaines caractéristiques sociodémographiques du lectorat du Devoir. La plus importante de ces caractéristiques est justement le «tirage» ou, plus exactement, le nombre d'exemplaires vendus, car il faut distinguer le nombre d'exemplaires imprimés de celui qui se rend jusqu'aux lecteurs. La comparaison entre les tirages de plusieurs journaux sera révélatrice de l'influence de chacun au sein de la population (ou des préférences du lectorat, pour prendre le point de vue inverse). Il faut aussi savoir qu'un exemplaire vendu est généralement lu par plus d'une personne, mais les statistiques historiques nous manquent sur ce point. Ce type d'analyse ressemble à la démarche d'un publicitaire chargé de sélectionner des médias pour une campagne. L'analyse portera d'abord sur l'ampleur de l'auditoire (du lectorat pour

19. Fernande Roy et Jean de Bonville, «La recherche sur l'histoire de la presse québécoise. Bilan et perspectives ", Recherches sociographiques, 41,1, (2000): 15-51 en ligne, http://id.erudit.org/iderudit/057 324ar.

20. Id.

21. Ibid., 41 et suivantes.

22. Paragraphe 5, dans Dominique Marquis, «L'histoire de la presse au Québec: état des lieux et pistes de recherche", dans Micheline Cambron et Stéphanie Danaux, dir., Nouveaux bilans, Publications, La recherche sur la presse: nouveaux bilans nationaux et internationaux, mis à jour le 09/11/2013, dans le site Médias 19, en ligne, www.medias19.org/index.php ?id=15556. 
un journal), puis sur sa composition selon l'âge et le genre, sur sa localisation géographique et sur des variables comme leur pouvoir d'achat ou leur position sociale.

Les études en communication médiatique utilisent aussi ces divers types de données, en particulier les recherches qui s’intéressent aux industries culturelles ou aux grands médias. Les études en communication et en histoire se distinguent de la recherche appliquée en publicité par l'intérêt qu'elles portent au contenu même du journal. Les études en communication utilisent souvent l'analyse de contenu, comme le fait aussi Jean de Bonville $^{23}$, alors que les historiens s'intéressent plus fréquemment aux événements rapportés dans les journaux, à la pensée ou à la biographie des journalistes. La discipline historique a accordé, entre autres, une grande attention aux combats politiques portés par les médias. Par exemple, Jean-Paul de Lagrave proposait en 1980 «l'histoire d'une liberté de pensée sans cesse entravée ${ }^{24}$ » qui s'appuyait sur Le Devoir et beaucoup d'autres journaux. Un an plus tard, Pierre Godin publie La lutte pour l'information. Histoire de la presse écrite au Québec ${ }^{25}$ où il écrit que l'histoire du journalisme "épouse à merveille les contours de l'histoire politique du Québec ${ }^{26}$ ». Plus près de nous, en 2008, dans le premier d'une série de livres sur la presse à Montréal, Jacques G. Ruelland adhère aussi à cette perspective en adoptant le titre 1776 Naissance de l'imprimerie et de la liberté d'expression à Montréal ${ }^{27}$.

La recherche historique s'est aussi intéressée aux données sur le lectorat de la presse, comme l'a fait notamment Jean de Bonville dans son œuvre magistrale, La presse québécoise de 1884 à 1914. Genèse d'un média de masse ${ }^{28}$. Dans cette analyse, de Bonville étudie le contexte d'émergence de la presse dite "d'information" ainsi que l'ensemble des conditions de son succès. Les données sur les tirages y sont commentées en profondeur. Nous nous plaçons, modestement, dans cette perspective. Cependant, de Bonville dresse le portrait d'une époque où Le Devoir n'apparaît qu'à la toute fin (1910). Pour une période ultérieure, une analyse comparative des tirages,

23. Jean de Bonville, Les quotidiens montréalais de 1945 à 1985 : morphologie et contenu (Québec, Institut québécois de recherche sur la culture, 1995).

24. Jean-Paul de Lagrave, L'histoire de l'information au Québec (Montréal, Les Éditions La Presse, 1980), 14.

25. Pierre Godin, La lutte pour l'information (Montréal, Le Jour Éditeur, 1981).

26. Ibid., quatrième de couverture.

27. Jacques G. Ruelland, dir., 1776 Naissance de l'imprimerie et de la liberté d'expression à Montréal (Montréal, Petit Musée de l'impression, 2008).

28. Jean de Bonville, La presse québécoise de 1884 à 1914. Genèse d'un média de masse (Québec, Les Presses de l’Université Laval, 1988). 
mais aussi des tarifs publicitaires, des principaux quotidiens du Québec entre 1945 et 1995 est contextualisée, dans le mémoire de maîtrise de Louis Jacob, Histoire de la presse quotidienne au Québec de 1945 à $1995^{29}$. On y constate que la position du Devoir est loin d'être idéale quant à son rapport aux annonceurs.

Ces différentes approches de l'histoire de la presse sont aussi présentes dans des monographies portant sur Le Devoir. Les approches adoptées sont parfois plutôt classiques (réalisées à partir du contenu du journal ou des biographies de ses artisans), parfois directement situées dans la nouvelle perspective appelée par Roy et de Bonville (la presse pour elle-même). On peut ainsi dire que l'histoire du journal est bien balisée. Parmi les travaux majeurs sur ce quotidien, signalons en particulier le récent numéro thématique de la revue Communication $^{30}$, dirigé par Jean Charron et Jean de Bonville, qui analyse justement l'institution pour elle-même sous de nombreux aspects, à l'occasion de son centième anniversaire. À l'occasion $\mathrm{du} 85^{\mathrm{e}}$ anniversaire, sous la direction de Robert Lahaise, Le Devoir reflet du Québec au $20^{e}$ siècle ${ }^{31}$ avait déjà fait un vaste tour d'horizon de la vie du journal. L'analyse de son contenu y occupait une grande place. En 1980, Pierre-Philippe Gingras avait déjà offert ce qu’il qualifie lui-même de "chronologie», soit un historique organisé autour de ses différents directeurs ${ }^{32}$. Le Devoir. Un siècle québécois, publié à l'occasion du centenaire du journal, mérite une mention particulière pour la richesse de ses illustrations et pour la variété des témoignages qui y sont récoltés ${ }^{33}$. Une biographie récente, Pourquoi j'ai fondé Le Devoir ${ }^{34}$ de Mario Cardinal, vient éclairer les actions et les motivations du fondateur. Ajoutons que le site Internet du Devoir offre aussi d'intéressants matériaux publiés à l'occasion de ses anniversaires marquants ${ }^{35}$.

Comme nous utiliserons abondamment les statistiques, une réserve méthodologique s'impose. Les données provenant du GRMJ sont issues de plusieurs sources qui peuvent s'appuyer sur des définitions différentes du tirage. Nous faisons entièrement confiance à l'autorité du GRMJ pour

29. Louis Jacob, Histoire de la presse quotidienne au Québec de 1945 à 1995, mémoire de maîtrise (sciences de la communication), Université de Montréal, 2004.

30. Jean Charron et Jean de Bonville, dir., "Cent ans dans la vie d'un journal », numéro thématique, Communication, 29, 2 (2012), en ligne, http:/ / communication.revues.org/2747.

31. Robert Lahaise, Le Devoir reflet du Québec au $20^{e}$ siècle (Ville LaSalle, Hurtubise HMH, 1994).

32. Pierre-Philippe Gingras, Le Devoir (Montréal, Libre Expression, 1985), 9.

33. J.-F. Nadeau, op. cit.

34. Mario Cardinal, Pourquoi j'ai fondé Le Devoir. Henri Bourassa et son temps (Montréal, Libre Expression, 2010).

35. Gilles Paré, «Le Devoir, un historique», site du Devoir, en ligne, www.ledevoir.com/le-devoir/ histoire [consulté le 16 novembre 2016]. 
Tableau I

Tirage du Devoir selon deux sources

\begin{tabular}{|c|c|c|}
\hline Année & GRMJ & Jacob \\
\hline 1945 & 21580 & 20621 \\
\hline 1950 & 19556 & 19470 \\
\hline 1955 & 24180 & 25586 \\
\hline 1960 & 31306 & 36022 \\
\hline 1965 & 46163 & 48284 \\
\hline 1970 & 36280 & 37913 \\
\hline 1975 & 31096 & 28425 \\
\hline 1980 & 40478 & 42404 \\
\hline 1985 & 30768 & 32905 \\
\hline 1990 & 28206 & 28206 \\
\hline 1995 & 28206 & 30343 \\
\hline
\end{tabular}

Sources: Groupe de recherche sur les mutations du journalisme (GRMJ), Université Laval et Louis Jacob, Histoire de la presse quotidienne au Québec de 1945 à 1995, mémoire de maîtrise (sciences de la communication), Université de Montréal, 2004, 58, données du CARD.

la sélection de ses bases de données et de ses sources au fil du temps. Mais, si on consulte les données du Canadian Advertising Rates and Data (CARD), on verra souvent des résultats légèrement différents de ceux du GRMJ. Le tableau 1 illustre les différences qui peuvent surgir en comparant les sources. On voit, par exemple, que les valeurs retenues par le GRMJ ne sont pas identiques à celles du $C A R D^{36}$, un instrument de travail courant pour les publicitaires. Le CARD cite souvent les données vérifiées par l'Audit Bureau of Circulation, une des autorités reconnues pour la vérification du tirage, mais ce n'est pas la seule. Heureusement, la corrélation entre les deux séries est très forte $\left(\mathrm{R}^{2}=0,96\right)$. La base de données du GRMJ demeure la référence si on souhaite comparer l'évolution des tirages des journaux sur une longue période.

\section{ANALYSE DU TIRAGE DES QUOTIDIENS MONTRÉALAIS}

Avant d'analyser l'évolution du tirage du quotidien Le Devoir, posons notre regard sur celui de l'ensemble des quotidiens montréalais en circulation entre 1854 et 2003 (Graphique 1). Nous ne considèrerons ici que les principaux quotidiens, soit ceux qui ont eu une longue durée de vie. On note 


\section{Graphique I}

Tirage cumulé des principaux quotidiens montréalais selon la langue (1 854-2002)

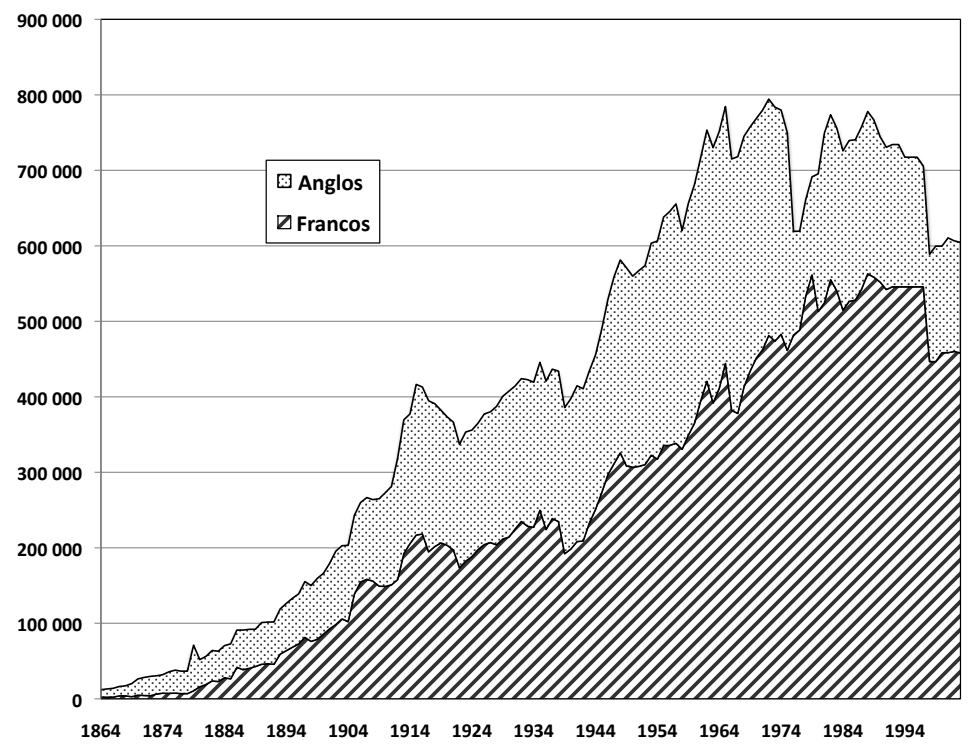

Source: Groupe de recherche sur les mutations du journalisme, Université Laval. Traitement et graphique par les auteurs.

que la croissance des tirages culmine dans la seconde moitié du $\mathrm{XX}^{\mathrm{e}}$ siècle. Ceux-ci amorcent, par la suite, une décroissance marquée par la disparition de trois titres majeurs, soit The Herald, le Montreal Star et le MontréalMatin. Au XX $\mathrm{X}^{\mathrm{e}}$ siècle, les quotidiens francophones représentent une part croissante du marché, qui reflète la composition linguistique du lectorat des journaux à Montréal.

L'analyse du tirage des principaux titres francophones (Graphique 2) montre que le marché des quotidiens rédigés en français est dominé par La Presse accompagnée, selon la période, par le Montréal-Matin ou le Journal de Montréal, de création plus récente. Ces deux derniers titres peuvent être classés dans la catégorie des journaux populaires de format tabloïd, alors que $\mathrm{La}$ Presse a connu un positionnement variable allant du populaire à une formule plutôt "grand public», toujours sur un papier de grand format (mais qui a récemment rétréci).

Selon nos données (voir le Graphique 3), Le Devoir aurait vendu 20000 exemplaires par jour en moyenne entre les années 1913 et 1916. Ce nombre doit être pris avec réserve, étant donné sa constance et sa belle 


\section{Graphique 2}

Tirage des principaux quotidiens montréalais publiés en français (1854-2002)

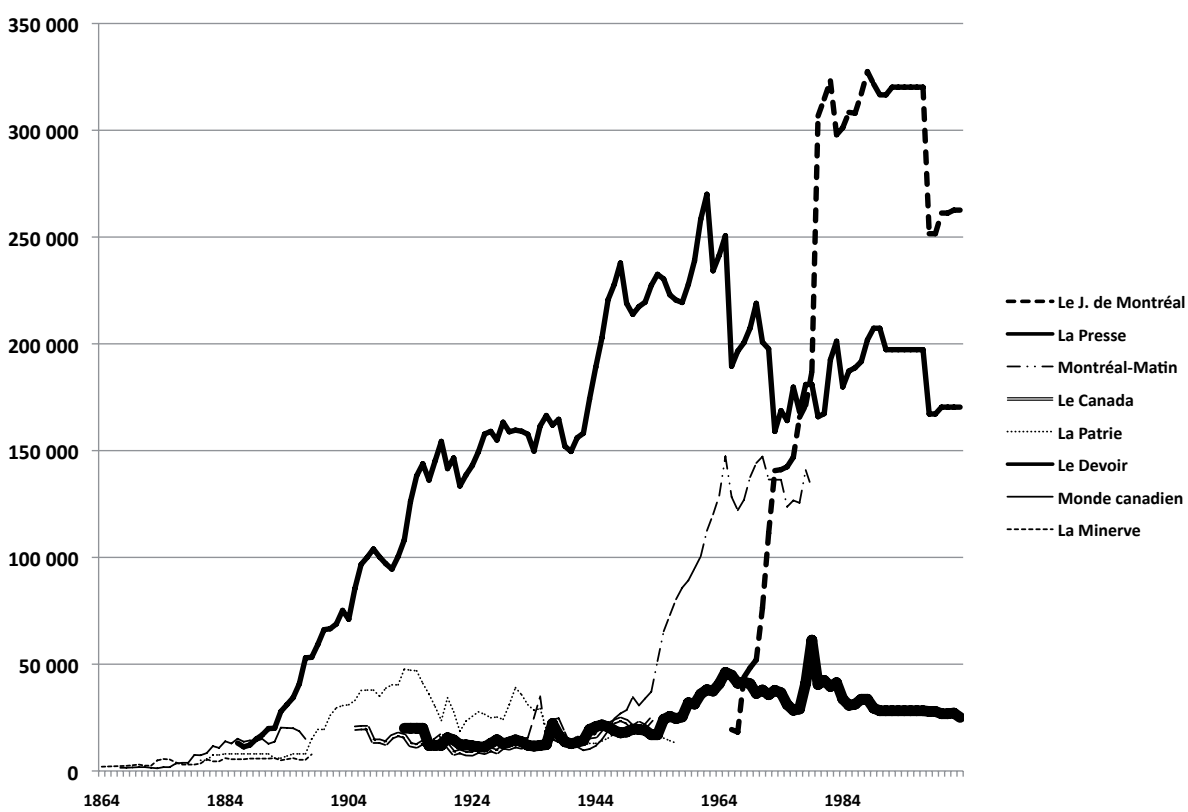

Source: Groupe de recherche sur les mutations du journalisme, Université Laval. Traitement et graphique par les auteurs.

rondeur. Par la suite, le tirage moyen annuel se situe entre 10000 et 15000 exemplaires par jour, selon les années. Dans les années 1940, le tirage passe le cap des 20000 exemplaires, puis celui des 30000 en 1959, à l'orée de la Révolution tranquille. Il dépasse les 40000 en 1964. N’oublions pas cependant que la population de Montréal et du Québec augmente aussi, ce qui explique en partie cette croissance. Mais ces années de fort tirage s'expliquent aussi par la conjoncture politique. Justement, un sommet est atteint en 1979 avec 61226 exemplaires vendus chaque jour, en moyenne. Le Parti Québécois prépare alors son premier référendum. Par la suite, le tirage baisse jusqu'aux environs de 25000 exemplaires par jour dans les années 2000.

D'un point de vue relatif, Le Devoir occupe une place qui fluctue entre $5 \%$ et $11 \%$ de l'ensemble du tirage des principaux quotidiens montréalais en français, soit, en moyenne, $7 \%$ (Graphique 4). On observe trois moments où sa part dépasse les $10 \%$ : en 1913, puis dans les années 1960 


\section{Graphique 3 \\ Tirage du journal Le Devoir (nombre d'exemplaires vendus quotidiennement entre 1913 et 2003)}

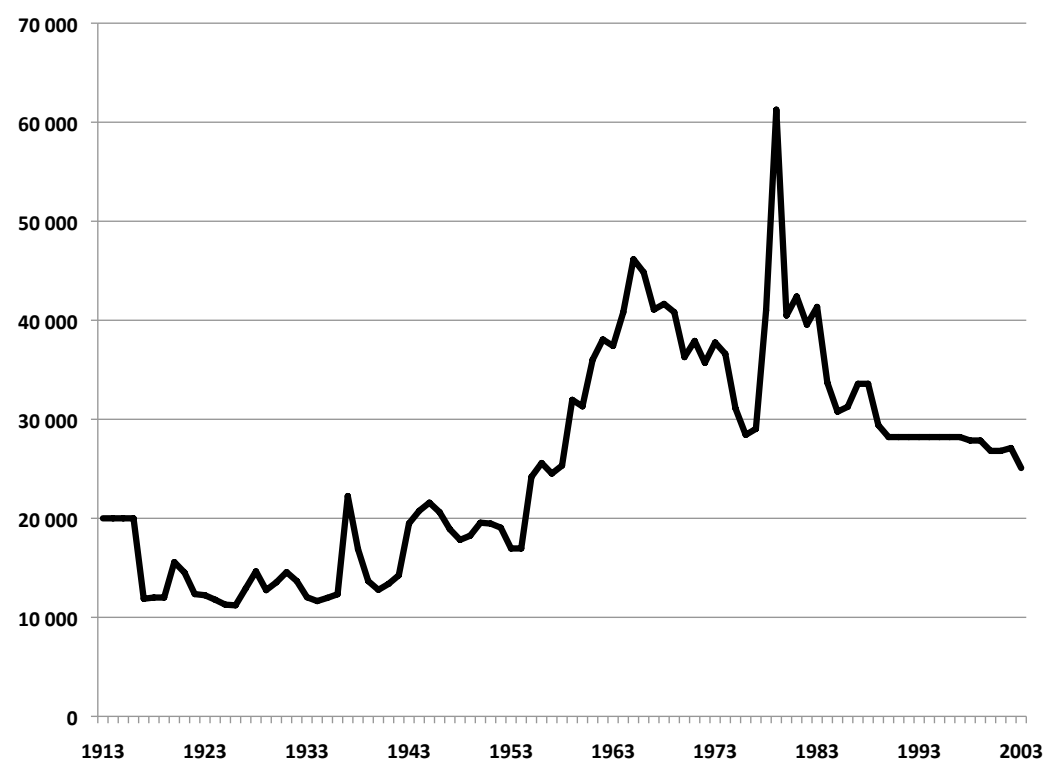

Source: Groupe de recherche sur les mutations du journalisme, Université Laval. Traitement et graphique par les auteurs.

et finalement, en 1979. La période qui s'ouvre avec les années 1980 marque une relative perte de part de marché. Mais, au total, Le Devoir réussit à conserver une place relativement stable, bien que modeste, dans l'ensemble des principaux quotidiens francophones de Montréal ${ }^{37}$. Cependant, comme nous le verrons plus loin, son influence sociale et politique dépasse clairement la simple valeur de son tirage.

\section{MOYENS DE DISTRIBUTION ET RÉPARTITION SELON LES RÉGIONS}

Qui donc forme ce lectorat? Pour répondre à cette question, nous analyserons, dans un premier temps, le nombre d'exemplaires selon le mode de distribution du journal (poste, commerçants, porteurs ${ }^{38}$ ) et ensuite, selon les régions où le journal est reçu.

37. Une analyse comprenant tous les quotidiens et non seulement ceux que nous avons identifiés comme les principaux pourrait nuancer cette affirmation.

38. Mode de distribution nord-américain amenant le journal à la porte de l'abonné en passant par un "porteur». 


\section{Graphique 4}

\section{Proportion (\%) du tirage des principaux quotidiens montréalais en français représentée par Le Devoir de 1913 à 2003}

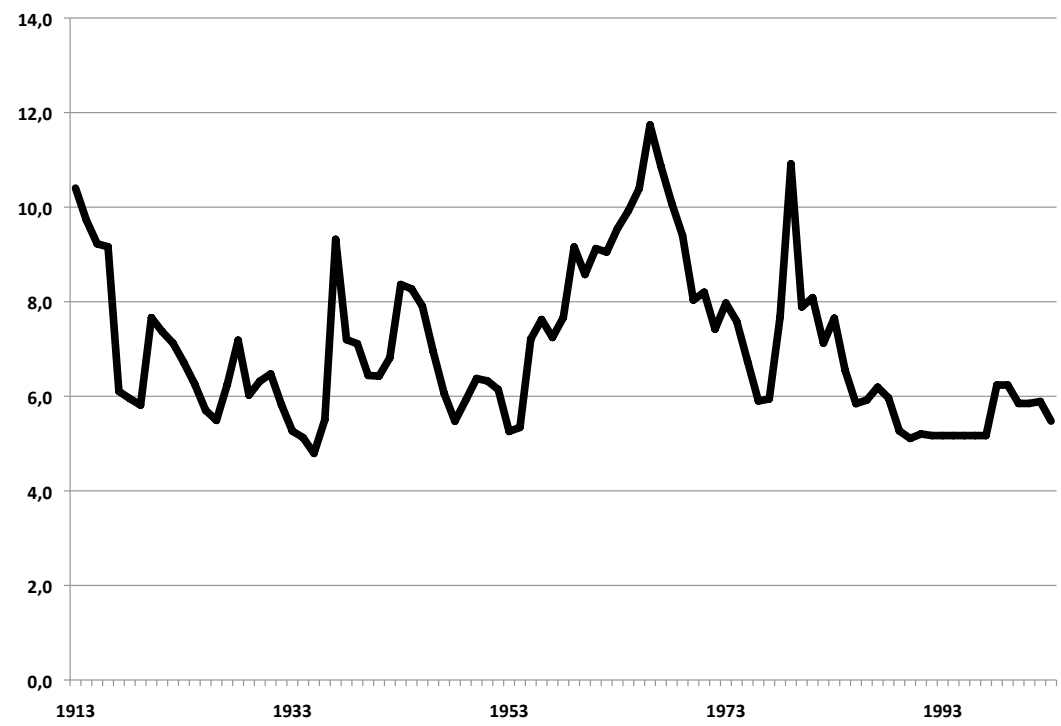

Source: Groupe de recherche sur les mutations du journalisme, Université Laval.Traitement et graphique par les auteurs.

Les données les plus complètes sur ce sujet sont celles de la distribution dite «brute» (ou gross distribution selon l'Audit Bureau of Circulation ${ }^{39}$ ). Nous disposons de ces données pour les années situées entre 1921 et 2001, mais seulement aux dix ans ${ }^{40}$. La distribution brute se distingue du nombre d'exemplaires vendus, car certains exemplaires sont distribués, mais non vendus. Cependant, en principe, les exemplaires distribués par la poste sont tous payés. Les quantités distribuées par la poste et celles vendues au domicile et en commerce sont assez proches jusqu'en 1971 où l'écart se creuse, alors que la proportion des exemplaires distribués par la poste diminue jusqu'à $22,9 \%$ (Graphique 5). Dix ans auparavant, cette proportion s'établissait à 49,7\%. En 1921, elle était de 58,2\%. Le Devoir commence donc sa vie d'abord distribué par la poste, comme beaucoup de journaux à l'époque, puis ce mode de distribution devient marginal vers

39. L'Audit Bureau of Circulation (ABC) est un organisme d'origine américaine qui, dès le début du $\mathrm{XX}^{\mathrm{e}}$ siècle, certifie les tirages des journaux pour l'usage des annonceurs.

40. Ces données sont disponibles pour les années se terminant par 1, sauf pour 1991 ici remplacé par 1992, car nous ne disposions pas de celles de 1991. 


\section{Graphique 5}

Proportion des exemplaires distribués par la poste (\%) entre 1921 et 1981

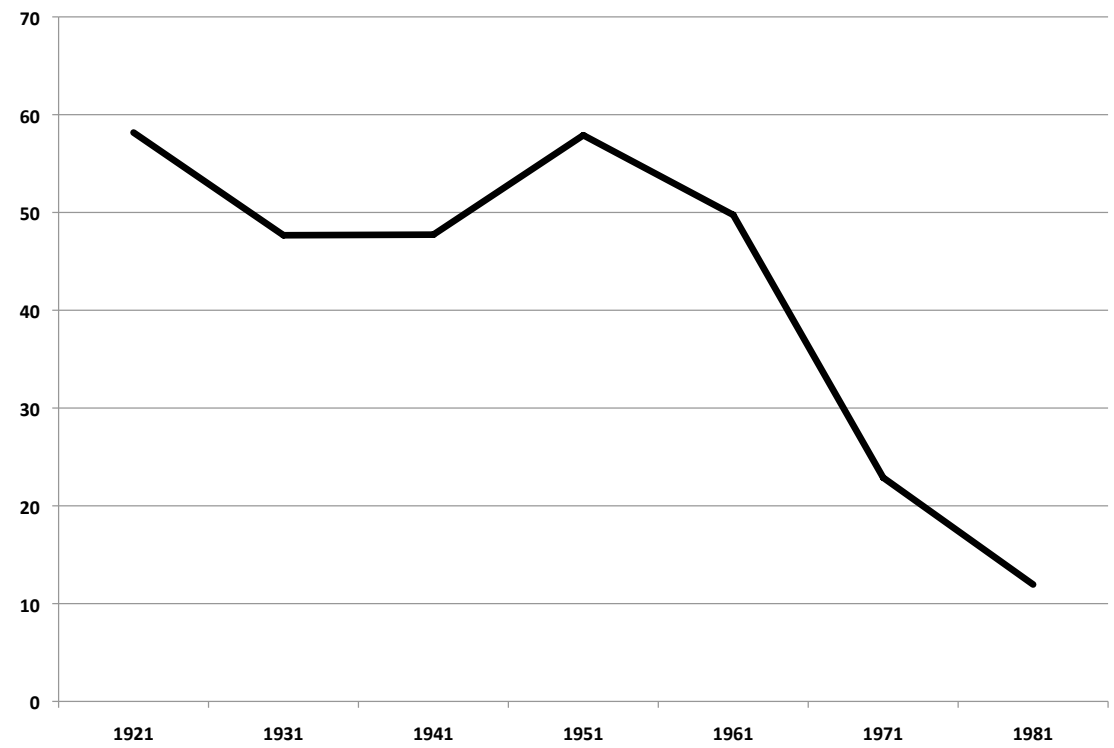

Source:Audit Bureau of Circulation, données fournies par le Groupe de recherche sur les mutations du journalisme, Université Laval. Traitement et graphique par les auteurs.

la fin du XX $X^{\mathrm{e}}$ siècle. En mars 2001, en semaine, le journal ne compte plus que 270 abonnés par envoi postal.

Les changements dans la distribution ne concernent pas uniquement la poste. En 1983, le journal confie sa distribution aux Messageries Dynamiques, une entreprise du groupe Quebecor ${ }^{41}$. Ce changement ajoute 3000 points de vente aux 4000 déjà rejoints et permet à un nombre accru d'abonnés de bénéficier de la livraison à domicile ${ }^{42}$. Ce changement suit un autre aussi important. Dans les années 1960, à la suite d'un bris mécanique des presses typographiques du journal, l'impression est confiée à l’Imprimerie Dumont qui utilise le procédé Offset ${ }^{43}$. En 1969, Quebecor

41. À noter qu'à cette époque, Quebecor s'écrit sans accent.

42. Auparavant, le service à domicile ne couvrait que «Montréal, certains quartiers de Québec et quelques centres régionaux", Le Devoir, 27 août 1983. On peut aussi ajouter que la fiabilité du service est améliorée, car le journal est distribué comme le Journal de Montréal.

43. Jean Dion, «Le Devoir, c'est moi - L'art de faire bonne impression», Le Devoir, 20 septembre 2010, en ligne: www.ledevoir.com/societe/medias / 296537 /le-devoir-c-est-moi-l-art-de-faire-bonne-impression [consulté le 20 octobre 2016]. 
achète l'imprimerie Dumont, débutant ainsi une longue relation d'affaires avec Le Devoir ${ }^{44}$. Un autre virage de la distribution se produira au tournant $\mathrm{du} \mathrm{XXI}^{\mathrm{e}}$ siècle avec la diffusion électronique par un site Web et, ensuite, par une application sur tablette électronique.

Revenons en 1921 où la distribution par la poste domine presque exclusivement pour les exemplaires expédiés à l'extérieur de la région de Montréal, sauf à Ottawa où 88 exemplaires sont postés alors que 50 sont vendus par des commerçants. Aux États-Unis, la ville de Manchester reçoit 28 exemplaires par la poste et Woonsocket, 45. Ce sont probablement les Canadiens français émigrés en ces lieux qui y sont abonnés. À Montréal même, les vendeurs et les porteurs (dealers $\&$ carriers) distribuent presque deux fois plus d'exemplaires que les vendeurs sur la rue (street vendors). En 1941, 402 exemplaires sont postés en Ontario, 273 le sont dans les autres provinces canadiennes, 218 aux États-Unis et 60 ailleurs dans le monde. En 1971, en Ontario, en semaine, les exemplaires distribués par des commerces ou des porteurs surpassent ceux qui sont postés (1460 contre 1270). La même année, 310 exemplaires sont postés aux États-Unis et 323 ailleurs dans le monde. En mars 1992, en semaine, 8837 exemplaires sont distribués par porteur dans la région de Montréal contre 5919 exemplaires vendus à l'unité. Seulement 312 exemplaires sont postés en semaine alors que 2551 exemplaires sont vendus en Ontario (dont 2257 à Ottawa), 155 dans les autres provinces, 94 aux États-Unis et 71 ailleurs dans le monde par ce mode de distribution. Tout au long du siècle donc, la région d'Ottawa prend une part croissante du lectorat du journal. Les États-Unis, qui ne représentent qu'une petite fraction du lectorat, connaissent une baisse, probablement subséquente à la décroissance des communautés canadiennes-françaises du nord-est. Par ailleurs, les abonnés qui reçoivent le journal par porteur y sont toujours proportionnellement nombreux, ce qui assure une certaine stabilité au journal et intéresse probablement certains annonceurs.

Regardons maintenant la distribution du journal selon les régions (Graphique 6). À l'intérieur du Québec, Montréal occupe évidemment une place particulière dans le lectorat du journal. Il faut cependant souligner que la définition de cette région varie dans le temps. Les limites géogra-

44. Daniel Coulombe et Jean Charron notent que «Pierre Péladeau, notamment, a renoncé à plus de 2 millions de dollars de dettes que l'Imprimerie populaire limitée avait contractées à l'endroit de l'Imprimerie Dumont, une filiale de Quebecor. », dans "L'évolution de la situation financière du Devoir à la lumière de ses états financiers ", Communication, 29, 2 (2012), en ligne, https: / / communication.revues. org/2798, paragraphe 29 . 


\section{Graphique 6}

Proportion de la distribution brute du journal Le Devoir selon l'aire géographique entre I92I et 1992

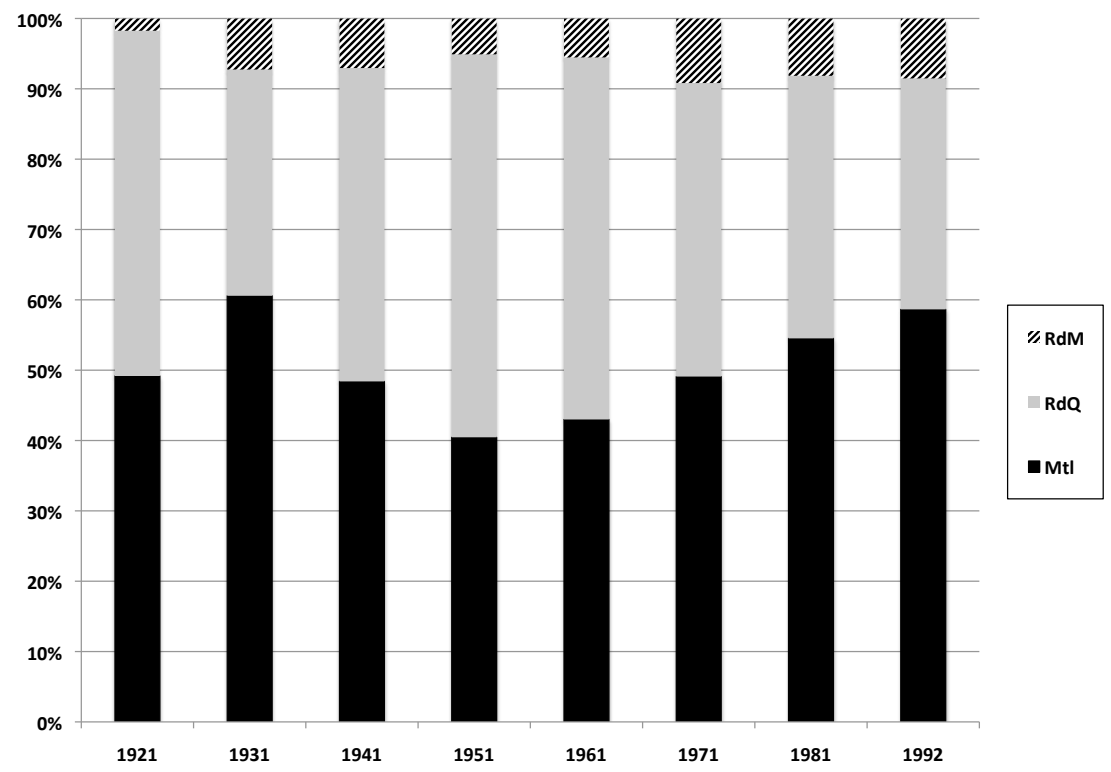

Mtl: région de Montréal: RdQ: reste du Québec; RdM: reste du monde

Source:Audit Bureau of Circulation, données fournies par le Groupe de recherche sur les mutations du journalisme, Université Laval. Traitement et graphique par les auteurs

phiques de la ville changent, car celle-ci et sa région sont en expansion. Le concept territorial du Audit Bureau of Circulation (ABC) se modifie aussi. En fin de période, la définition de la région montréalaise change tellement qu'il devient très difficile de raccorder les données. Notons que l'ABC s'appuie sur une notion économique de la ville, soit la zone étendue de commerce centrée sur Montréal, plutôt que sur une notion juridique. Aux fins de la présente analyse, nous ne pouvons pas mesurer la part d'une entité géographique aux limites fixes, puisque nous devons tenir compte de l'évolution des contours de la région.

Les données de la distribution brute entre 1921 et 1992 révèlent que la région de Montréal reçoit entre 40 et $60 \%$ des exemplaires distribués, alors que la population de la ville (selon ses limites de 2011) ne représente qu'entre 33 et $23 \%$ de celle du Québec ${ }^{45}$. Cela n'empêche pas le journal de

45. Soulignons encore que les définitions de la région par ABC et selon les limites de la ville ne concordent pas. Les populations comparées de Montréal (limites de 2011) et du Québec sont calculées 
se présenter comme un organe "national» étant distribué dans toute la province et même dans le reste du Canada français. Notons qu'il s'agit là d'une définition minimaliste de cette notion, car il faudrait aussi analyser le contenu du journal pour confirmer ou non ses prétentions "nationales». En 1931, la ville de Québec et sa région reçoivent 522 exemplaires, ce qui est très peu comparé à Montréal (7852). La part des régions est à un sommet en 1951, Québec reçoit alors 1082 exemplaires, 535 exemplaires partent pour Chicoutimi, 456 exemplaires sont expédiés dans le comté de Saint-Maurice (Shawinigan), 324 à Sherbrooke, 307 en Abitibi (comté) et 83 dans le comté de Matane. Dix ans plus tard, en 1961, Québec reçoit 3911 exemplaires, puis 5584 en 1971, mais seulement 3880 en 1991. Selon notre analyse, ces données permettent de conclure en une influence réelle ou, inversement, du point de vue du lectorat, attester d'un intérêt largement répandu au niveau provincial pour le journal (Graphique 6).

\section{ANALYSE DES CARACTÉRISTIQUES DU LECTORAT}

Mais de quels types de lecteurs parlons-nous? Nous venons de voir qu'il ne s'agit pas d'un lectorat massif, en comparaison avec les quotidiens les plus populaires à chaque époque. Toutefois, la proportion de lecteurs du journal Le Devoir parmi le lectorat des quotidiens québécois francophones demeure plutôt stable, avec une forte composante hors de Montréal.

\section{Les lectures de l'élite canadienne-française}

Une enquête réalisée en 1942 ouvre une petite fenêtre sur les caractéristiques de ce lectorat ${ }^{46}$. Il s'agit d'un sondage par questionnaire réalisé par l'auteur et éditeur Albert Lévesque, formé en droit et administrateur de la Ligue d'Action française. Ce dernier cherchait à «démontrer que les 3,000,000 de Canadiens de langue française constituent, au sens moderne du terme, une $\mathrm{NATION}^{47}$ ». Ayant pour but d'analyser le «comportement familial» des Canadiens français, l'enquête portait sur un important échantillon non aléatoire de 2229 familles issues de l'«élite ou classe moyenne supérieure ${ }^{48}$ du Canada français. Cette élite se définissait par l'activité professionnelle du père (cultivateur, homme d'affaires, homme de métier, professionnel). La répartition régionale va comme suit: $36 \%$

par l'Institut de la Statistique du Québec, www.stat.gouv.qc.ca/docs-hmi/statistiques/population-demographie/structure/Tableau_top_10.htm, [consulté le 4 novembre 2016].

46. Nous devons cette trouvaille à notre collègue Denis Saint-Jacques de l'Université Laval.

47. Albert Lévesque, "Entrez-donc": analyse du comportement familial de langue française au Canada, Première étape. Sentiments et intérêts de l'élite (Montréal, Les Informations Albert Lévesque, 1944), 11.

48. Selon l'expression utilisée dans ibid. 
dans la région de Montréal, $40 \%$ dans le reste du Québec, $12 \%$ en Ontario et $12 \%$ dans les Maritimes. Les questions portent, entre autres, sur l'usage des médias et, en particulier, sur la lecture des quotidiens. Voici donc l'ancêtre des enquêtes contemporaines sur les comportements culturels!

Sans surprise, on retrouve Le Devoir parmi les quotidiens mentionnés dans l'enquête. La mesure présentée est la proportion de l'échantillon qui a «l'habitude de lire» chacun des titres (soit la portée en langage technique contemporain, ou encore, l'équivalent de la cote d'écoute en télévision) et cette proportion est détaillée selon la position dans la famille, la profession du père (Illustration 1) et la région. L'Action catholique et La Presse dominent pour la portée (avec respectivement $34 \%$ et $28 \%$ de pénétration dans cette «élite»). On sera peut-être surpris de retrouver L'Action catholique ici, mais il faut savoir que ce journal avait, en 1950, un tirage vendu de plus de 78000 exemplaires $^{49}$. Selon Bibliothèque et Archives nationales du Québec (BAnQ), «[d]iffusé dans la grande région de Québec, L’Action catholique a été longtemps le quotidien préféré des ruraux ${ }^{50} \ldots$ ». On voit aussi ici l'effet de la sélection élitiste des répondants dans cette enquête. Le Devoir, pour sa part, bénéficie d'une portée de $15 \%$, soit un peu plus de la moitié de celle de La Presse.

C'est sur le plan de la composition du lectorat du journal Le Devoir que les données du sondage de 1942 se révèlent les plus intéressantes. On observe que ce sont les professionnels qui lisent le plus Le Devoir (30\%), mais qu'ils lisent encore plus La Presse (40\%). Les cultivateurs ne sont lecteurs du quotidien Le Devoir que dans une proportion de $9 \%$ contre $42 \%$ qui lisent L'Action catholique. Les hommes d'affaires lisent plus Le Devoir que la moyenne de l'élite (15\%). À l'intérieur de la famille ${ }^{51}$, Le Devoir est davantage le journal du père $(23 \%)$ que celui de la mère (13\%). Parallèlement, le fils le lit plus (17\%) que la fille (9\%). C'est dans la ville de Montréal que Le Devoir touche sa plus forte proportion de l'élite, $34 \%$ contre $12 \%$ pour le reste du Québec et $8 \%$ dans les Maritimes. Il n'est donc pas faux, selon ces données, de dire que Le Devoir est, en 1942, le journal d’une élite, mais que ce n'est pas le seul journal de l'élite canadienne-française.

Si Le Devoir est le journal d'une certaine élite, il rejoint aussi d'autres lecteurs. Dans une lettre datée du 4 mars $1954^{52}$, Gérard Filion, alors

49. L. Jacob, op. cit., 104.

50. Bibliothèque et Archives nationales Québec, L'Action catholique : organe de l'Action sociale catholique, lien Pour en savoir plus..., http:/ / collections.banq.qc.ca/ark:/ $52327 / 357283$ [consulté le 2 février 2010].

51. A. Lévesque, op. cit., 125.

52. Gérard Filion, (Lettre à G.E. Patry), 1 f., tiré des archives du Devoir par le GRMJ, 4 mars 1954. 


\section{Illustration I}

Enquête de 1942 auprès de l'uélite " canadienne-française

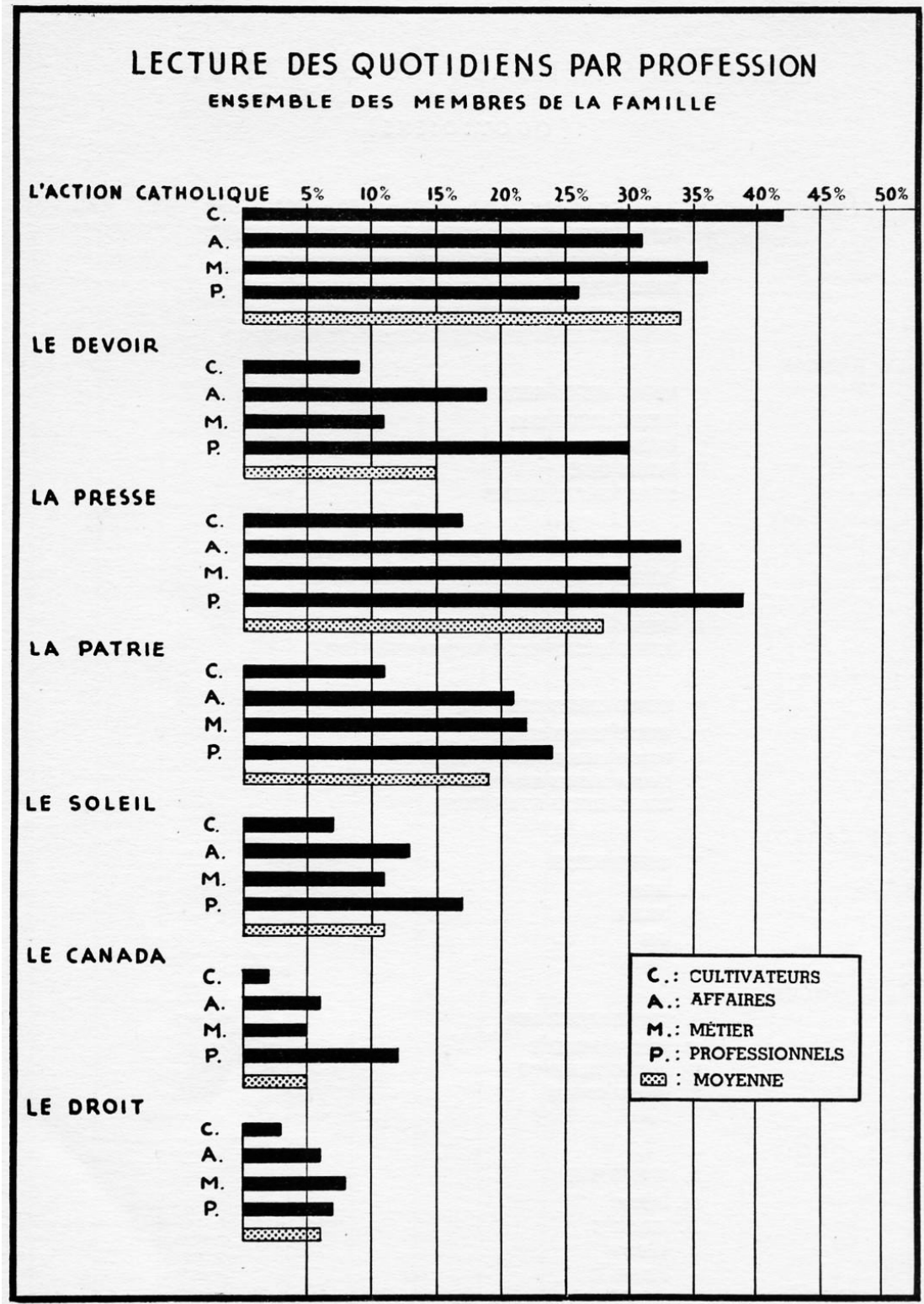

Source:Albert Lévesque, «Entrez-donc»: analyse du comportement familial de langue française au Canada, Première étape. Sentiments et intérêts de l'élite (Montréal, Les Informations Albert Lévesque, 1944), I44. 
directeur du quotidien, remercie G. E. Patry, président du Syndicat catholique des travailleurs du meuble de Victoriaville, pour l'abonnement de tous les officiers du syndicat. "L'attitude que Le Devoir a tenue depuis quelques années sur la plupart des problèmes sociaux lui a fait perdre de précieux appuis dans la classe bourgeoise ", écrit Filion. On peut sourire devant cette dénonciation de la bourgeoisie en pensant aux fonctions qu'occupera ensuite Filion! Mais on peut constater que parmi les lecteurs $\mathrm{du}$ journal, on trouve ceux qui organisent un mouvement ouvrier qui, justement, se retournera contre certains éléments de l'élite canadiennefrançaise d'alors.

\section{Un sondage auprès des abonnés}

Tournons-nous maintenant vers des sondages réalisés pour le compte du journal. La première enquête fut réalisée par Edsall Research Limited (Marketing Consultants) en 1964 auprès de 509 abonnés. Claude Ryan, alors directeur du quotidien, est impliqué dans l'opération. Les abonnés ont probablement des caractéristiques différentes des autres lecteurs, mais il s'agit de personnes relativement faciles à rejoindre. Par ailleurs, les annonceurs sont particulièrement intéressés par les abonnés, un groupe probablement plus prospère que les autres lecteurs. Visiblement, les responsables de l'enquête souhaitent démontrer la valeur particulière du lectorat du journal. Le sondage vise à connaître l'occupation et même "l'influence sociale exercée par le chef de famille ${ }^{53}$ ».

Une analyse préliminaire de ces 509 noms a révélé la présence de 39 médecins et chirurgiens et de 64 membres du clergé (prêtres séculiers, religieux et religieuses). En projetant ces chiffres à la totalité des abonnés du Devoir, nous pouvons conclure qu'environ 2,000 médecins et chirurgiens [...] et 3,200 membres du clergé sont abonnés au Devoir ${ }^{54}$.

Quelle force de frappe en effet! La moitié des répondants ont un diplôme universitaire, $26 \%$ sont allés en Europe au cours des trois dernières années et $56 \%$ ont pris l'avion. L'illustration 2 montre l'occupation du chef de famille chez les abonnés sondés. La première ligne indique que $11 \%$ sont des membres du clergé. Ce positionnement des fonctions ecclésiastiques n'est évidemment pas fortuit. Dans ce tableau (présenté conformément à sa présentation dans le rapport), l'ordre des occupations n'est ni alphabétique ni décroissant. Il reflète vraisemblablement l'importance sociale des

53. GRMJ, Archives du Devoir, Edsall Research Limited, «Etude (sic) du profil des lecteurs du Devoir», 1965, 10 f. + ann., copie numérisée,.

54. Id. 


\section{Illustration 2}

\section{Enquête de I 964 auprès des abonnés}

D. Occupation du chef de famille

Occupation
Membres du clergé
Médecins, chirurgiens, vétérinaires,
dentistes, optométristes
Avocats et notaires
Ingénieurs conseils, architectes,
chimistes, etc.
Chefs d'entreprise, administrateurs
et commerçants
Professeurs laics
Contremałtres, surveillants, etc.
Vendeurs (excluant les commis
préposés a la vente)
Collets blancs (y compris les services)
Collets bleus spécialisés
Collets bleus non spécialisés
Pensionnés
Etudiants
Pas de réponse

\section{$\%$ des réponses}

$11 \%$

$8 \%$

$5 \%$

$1 \%$

$12 \%$

$12 \%$

$4 \%$

$1 \%$

$23 \%$

$7 \%$

$1 \%$

$4 \%$

$1 \%$

$10 \%$

Source: Archive du Devoir, numérisée et fournie par le Groupe de recherche sur les mutations du journalisme, Université Laval.

occupations (clergé, médecins, avocats, ingénieurs...) aux yeux des responsables du quotidien. Cette caractéristique du lectorat remonte à la fondation du journal qui s'était donné comme cause, entre autres, «la défense de la foi catholique ${ }^{55}$ ", comme d'autres journaux à l'époque. D'un autre point de vue, soulignons que la proportion des étudiants serait plus importante si les données comprenaient l'ensemble du lectorat plutôt que seulement ses abonnés, d'autant plus que le corps enseignant des collèges recommandait la lecture du Devoir ${ }^{56}$.

À la fin du rapport, on trouve des commentaires des répondants recueillis lors de l'enquête. Ceux-ci diffèrent. Certains indiquent qu'ils pensent que le journal a des tendances anticléricales (en 1964, le journal a changé!), d'autres que les pages sportives sont trop brèves, etc.

55. Martin David-Blais, Guy Marchessault et Stanisław Sokołowski, «Les lecteurs catholiques québécois sont-ils demeurés fidèles au quotidien Le Devoir après les changements d'orientation des années 1990 ?», Communication, 29, 2 (2012), en ligne, http:/ / communication.revues.org/2774.

56. Et aussi, sans doute, des universités. Mais nous n’avons pas de témoignage sous la main pour le prouver. 


\section{Deux sondages pour intéresser les annonceurs}

La seconde enquête dont nous disposons date de 1975, mais la source n'apparait pas sur la copie utilisée $e^{57}$. Plusieurs pages manquent ou sont illisibles. Elle se présente comme un sondage presque exclusivement orienté sur les dépenses de consommation des lecteurs. Ici et là, quelques bribes révèlent des caractéristiques intéressantes. Les universitaires dominent (63\% des répondants), alors que $71 \%$ des lecteurs et $61 \%$ des lectrices lisent Le Devoir tous les jours. «Les postes CBF-AM et FM sont les stations de radio les plus écoutées par les répondants ${ }^{58}$.» Il s'agit des stations de Radio-Canada, une corrélation peu surprenante. Par ailleurs, «Les vins importés étaient préférés par quatre-vingt-quatre pour-cent (84\%) des lecteurs ${ }^{59}$.» Bref, le lectorat du quotidien Le Devoir fait partie des strates supérieures de la société.

Regardons maintenant le sondage très professionnel réalisé par Jean Jolicœur et Associés en 1984, encore auprès des abonnées du quotidien. Notons qu'une enquête semblable avait été réalisée l'année précédente. Ce sondage a été réalisé à partir d'un échantillon aléatoire de 641 abonnés. On y trouve une analyse détaillée des caractéristiques des répondants. L'enquête insiste sur l'appréciation de la section économique du journal ainsi que sur la consommation culturelle des abonnés. Ceux-ci sont surtout des hommes (72\%) et le groupe des 35 à 64 ans y est proportionnellement plus important que dans l'ensemble de la population du Québec, l'écart étant encore plus marqué pour la tranche d'âge des 35 à 44 ans :

Les abonnés constituent un groupe ayant en général des revenus élevés, un haut niveau de scolarité et une position occupationnelle caractérisée par des fonctions de gestion. [...] D’autre part, les gestionnaires se caractérisent aussi par une forte compétence culturelle ${ }^{60}$.

Une forte proportion des abonnés ( $83 \%$ ) détient un diplôme universitaire, même que $21 \%$ ont un doctorat (incluant possiblement un doctorat en médecine, ce qui pourrait être interprété autrement). Les diplômes le sont d'abord en sciences sociales (33\%) et en gestion (14\%). Les abonnés sont alors, pour plus de la moitié, à l'emploi du secteur public ou parapublic (Illustration 3). Les deux tiers sont allés au cinéma dans les trois derniers mois, $54 \%$ ont assisté à au moins un spectacle, ce qui les situe

57. GRMJ, Archives du Devoir, Auteur inconnu, «Sondage auprès des lecteurs du "Devoir” ", copie numérisée incomplète, non paginée, 1975.

58. Id.

59. Id.

60. GRMJ, Archives du Devoir, Jolicoeur, Jean et Associés. «Étude de la clientèle», copie numérisée, 29 f. + ann, 1964 


\section{Illustration 3}

\section{Occupation des abonnés en 1983 et 1984}

Secteurs d'activités économiques

$\begin{array}{lcc} & \frac{1984}{(100)} & \frac{1983}{(100)} \\ & \% & \% \\ \text { Para-public } & 34 & 37 \\ \text { Public } & 19 & 16 \\ \text { Services professionnels } & 15 & 13 \\ \text { Média et communication } & 9 & 6 \\ \text { Fabrication, transport } & 6 & 7 \\ \text { Services financiers } & 5 & 8 \\ \text { Distribution, commerce au détail } & 5 & 3 \\ \text { Services personnels } & & 4 \\ \text { Autres } & 6 & 6\end{array}$

Source:Jolicoeur et Ass., archive du Devoir, numérisée et fournie par le Groupe de recherche sur les mutations du journalisme, Université Laval.

comme d'importants consommateurs de biens culturels. De plus, « $40 \%$ d'entre eux dépens[ent] plus de $30 \$$ par mois en vins ${ }^{61} »$. Toujours en 1984 , $43 \%$ des abonnés lisent aussi La Presse et $28 \%$ sont abonnés au journal Les Affaires.

Les commentaires libres des abonnés ne manquent pas d’intérêt: «Vous respectez mal l'esprit nationaliste du fondateur. La couverture "Religion" s'est détériorée surtout depuis deux ans ${ }^{62}$.» Un autre affirme qu' "il est devenu un journal très ordinaire depuis le départ de M. Filion ${ }^{63}$ ». "J'aimerais tous les jours avoir un cahier sur les arts, les spectacles, les films avec des images à l'appui ${ }^{64}$.» Pourtant, «[l]a très grande majorité des abonnés (94\%) se déclarent satisfaits de la facture générale du journal et $70 \%$ considèrent que le journal s'est amélioré depuis douze mois ${ }^{65} \%$.

Toutes ces enquêtes peuvent nous laisser sur notre faim si nous voulons tracer un portrait nuancé du lectorat du Devoir. Celle de Lévesque utilise un mode d'échantillonnage plutôt typique des enquêtes qualitatives. Les

\footnotetext{
61. Ibid., p. 13 .

62. Ibid., dans l'Annexe, non paginée.

63. Id.

64. Id.

65. Id.
} 
sondages commandés par le Devoir ne rejoignent pas l'ensemble du lectorat (sans doute par souci d'économie) et ils sont motivés par le besoin de démontrer aux annonceurs l'importance économique et sociale de son lectorat. Ces données permettent toutefois de conclure que Le Devoir rejoint une proportion incontournable des classes économiquement favorisées et capables de participer aux orientations de la société canadienne-française et québécoise.

\section{CONCLUSION}

Le quotidien Le Devoir occupe une place relativement stable parmi les journaux québécois. Fondé au moment où la presse populaire prend son envol, il profite peu de la forte croissance des tirages des journaux qui caractérise la première moitié du $\mathrm{XX}^{\mathrm{e}}$ siècle. Cependant, le tirage de ce journal connaît une période faste pendant les années 1960 et 1970, une période qui a vu, entre autres, l'accomplissement de la Révolution tranquille, la fondation du Parti Québécois et le déroulement de la «crise d’octobre» de 1970. Au fil des années, Le Devoir représente une proportion relativement stable du tirage total des quotidiens francophones de Montréal. La distribution du journal par la poste est importante au début du $\mathrm{XX}^{\mathrm{e}}$ siècle et elle comprend bon nombre d'abonnés hors Québec. Mais ce phénomène s'estompe avec les années. La distribution au Québec hors de Montréal demeure assez importante pour conférer au Devoir une influence nationale.

Certaines archives permettent de tracer un portrait du lectorat du journal, mais leur petit nombre impose une réserve. Il en ressort clairement que son lectorat se retrouve en bonne partie parmi l'élite canadienne-française ou québécoise. Cette particularité est d’ailleurs revendiquée dans les conclusions des études sur les abonnés, car les annonceurs sont intéressés par un lectorat en mesure de dépenser. Mais il y a certainement une portion du lectorat qui ne correspond pas à cette image. La lettre de Filion, que nous avons évoquée précédemment, pointe dans cette direction. Qu'en est-il de la jeunesse étudiante et des autres fractions de la société? D’autres recherches seraient donc nécessaires pour mieux comprendre les motivations du lectorat du Devoir.

Qu'est-ce qui explique l'influence du Devoir, considérant son tirage limité? Nous répondrons en utilisant un modèle élaboré par le groupe de recherches de l'Université Laval sur les best-sellers, ces livres qui connaissent le succès en librairie ${ }^{66}$. Le succès d'un livre résulte d'une communi-

66. D. Saint-Jacques, J. Lemieux, C. Martin et V. Nadeau, op. cit. 
cation réussie entre un auteur, avec son éditeur, et un lectorat qui, règle générale, connaît et apprécie l'auteur. Cette communication prend la forme d'un texte qui fournit aux lecteurs et lectrices des outils pour comprendre leur propre vie et, aussi, pour élargir leur encyclopédie personnelle. À sa fondation, Le Devoir, annonce qu'il «appuiera les honnêtes gens et dénoncera les coquins » et il maintiendra cette ligne jusqu'à nos jours, avec de nombreuses adaptations. Plus exactement, il fournira à son lectorat un contenu qui correspond à ses besoins particuliers, qui va nourrir sa "distinction» pourrait-on dire à la suite de Pierre Bourdieu. Ce qui explique l'influence du journal, c'est sa façon de faire du journalisme «de qualité» alliée aux besoins d'une fraction relativement puissante de la population québécoise. Cette fraction viendra d'ailleurs au secours du journal dans les moments difficiles. Le Devoir n'est pas le seul média à s'adresser à ce public, mais il le fait d'une façon différente, «indépendante» diront plusieurs, appréciée de son public.

Aujourd'hui, les questions quant à la survie du journal se posent encore plus avec la numérisation des médias d'information et les nouvelles menaces qui pèsent sur le financement du journalisme par la publicité. Il demeure pertinent de s'intéresser au lectorat des journaux pour mieux comprendre les besoins et les attentes des lecteurs. L'exercice d'analyse statistique que nous avons réalisé ici pourrait s’appliquer aux nombreuses autres institutions qui ont marqué l'histoire de la presse québécoise. Les bases de données, comme celle du GRMJ, offrent un riche matériel à exploiter. Il serait aussi pertinent de poursuivre les travaux de Jacob ${ }^{67}$ pour les années ultérieures à 1995. Enfin, tout comme le mentionnaient Roy et de Bonville ${ }^{68}$, il nous semble nécessaire d'inviter les historiens à s'intéresser davantage aux journaux en tant qu'institutions historiques et de considérer leurs spécificités économiques et politiques, mais aussi les caractéristiques du lectorat, pour mieux mesurer leur apport à l'histoire du pays. 